References

[1] Dunham Jackson, The theory of approximation, vol. XI, A. M. S. Pub., New York.

[2] P. P. Korovkin, Linear operators and approximation theory, India.

[3] S. M. Lozinski, On convergence and summability of Fourier series and interpolation processes, Math. Sbornik 14 (56) (1944), pp. 175-268.

[4] I. P. Natanson, Constructive function theory, New York.

[5] A. Sharma and A. K. Varma, Trigonometric interpolation Duke Math. J., vol. 32, No. 2 (1965), pp. 341-350.

[6] 0 . Shisha and B. Mond, The degree of approximation to periodic functions by linear positive operators, Jour. of Approximation Theory I (1968), pp. 335-339.

[7] A. K. Varma. Simultaneous approximation of periodic continuous functions and their derivative, Israel J. Math. 6 (1968), pp. 67-79.

[8] A. Zygmund, Trigonometric series, vol. II, New York 1959.

\section{Fractional powers of operators and Bessel potentials on Hilbert space}

by

MICHAEL J. FISHER* (Missoula, Montana)

Abstract. Two candidates for the title "the Bessel potential" over a real separable Hilbert space are studied with the theory of fractional powers of operators and shown to define equivalent Sobolev spaces $L_{p}^{\alpha}(H) . L_{p}^{\alpha}(H)$ is shown to be equivalent to $D\left(T^{\alpha}\right)$ when $(-T)$ is the infinitesmal generator of the Poisson integral and when $D\left(T^{\alpha}\right)$ is equipped with the graph norm. The Bessel potentials of purely imaginary order are shown to be bounded on the reflexive $L_{p}(H)$ and to form a strongly continuous boundary value group for the Bessel potentials $J^{\alpha}$ with $\operatorname{Re}(\alpha)>0$.

Introduction. In [3] we defined the Bessel potential over a real separable Hilbert space, $H$, and studied the family of singular integral operators $G^{\alpha}: L_{p}^{\alpha}(H) \rightarrow L_{p}(H)$, where $L_{p}^{\alpha}(H)$ is the image of $L_{p}(H)$ under the Bessel potential $J^{\alpha} \cdot J^{\alpha}(f)=\Gamma(\alpha)^{-1} \int_{0}^{\infty} P_{t}(f) t^{\alpha-1} e^{-t} d t$, where $P_{t}(f)$ is the Poisson integral of $f$; [2]. The norm in $L_{p}^{a}(H)$ is $\|g\|_{\alpha, p}=\|f\|_{p}$ when $g=J^{\alpha}(f)$. The purpose of this paper is to examine the Bessel potential operators more closely than they were studied in [3]. Specifically, we shall examine two prominent candidates for the designation of "the Bessel potential" over an infinite dimensional Hilbert space and show that the spaces $L_{p}^{a}(H)$ defined using these operators are equivalent to the domain of a certain closed densely defined operator when this domain is equipped with the graph norm. Secondly, we shall examine the semigroup $J^{\alpha}$ in $\operatorname{Re}(\alpha) \geqslant 0$ and show that the boundary values, $J^{i \gamma}$, form a strongly continuous group of bounded operators on $L_{p}(H)$ if $1<p<\infty$. The paper closes with a discussion of the infinitesmal generators of $J^{\beta}$, $\beta>0$, and $J^{i \gamma}$

Throughout this paper $K, K(\alpha), K(p, \alpha)$ etc. $(M, M(\alpha), M(p, \alpha)$, etc.) denote positive (complex) constants which depend only on the parameters shown. If $T$ is a linear operator on a Banach space $X, D(T)$ denotes the domain of $T$ and $R(T)$ denotes the range of $T$.

* Research supported in part by the National Science Foundation grants NSF-GP-8839 and NSF-GP-24574. 
1. Definitions and Preliminaries. Let $H$ be a real separable Hilbert space, let $n_{t}$ denote the weak normal distribution on $H$ which has variance parameter $t / 2$ and is centered at the origin in $H$, and let $B$ be a HilbertSchmidt operator on $H$. Let $n=n_{2}, L_{p}(H)=L_{p}(H, n)$ be the Banach space of $p$-power integrable complex valued functions on $H$, and let $y \rightarrow T_{y}$ denote the regular representation of the additive group of $H$ acting on $L_{p}(H)$. See Section 1 of [2] for the measure theoretic preliminaries. Define

$$
H_{t}(f)=\int_{H} T_{y} f d n_{t} \circ B^{-1}(y) \quad \text { and } \quad P_{z}(f)=\int_{0}^{\infty} H_{t}(f) N_{t}(z) d t / t,
$$

where $N_{t}(z)=(\pi t)^{-1 / 2} z \exp \left(-t^{-1} z^{2}\right) . P_{z}(f)$ is the Poisson integral of $f$. The strongly continuous contraction semi-groups $H_{t}$ and $P_{z}$ were studied in $[2] ; P_{z}$ extends as an analytic semi-group to $|\arg (z)|<\pi / 4$.

Set

$$
J_{1}^{\alpha}(f)=\Gamma(\alpha)^{-1} \int_{0}^{\infty} P_{t}(f) t^{\alpha-1} e^{-t} d t
$$

and

$$
J_{2}^{\alpha}(f)=\Gamma(\alpha / 2)^{-1} \int_{0}^{\infty} H_{t}(f) t^{(\alpha-2) / 2} e^{-t} d t .
$$

In the nomenclature of Komatsu's theory of fractional powers of operators $[4], J_{1}^{\alpha}(f)=(1+T)^{-a} f$ and $J_{2}^{\alpha}(f)=\left(1+T^{2}\right)^{-\alpha / 2}$, where $P_{z}(f)=\exp (-z T) f$ We shall show in Section 3 that when $\alpha>0$, the $\left(J_{i}^{\alpha}\right)^{-1} J_{l_{c}}^{\alpha}, i, k=1,2$, are bounded operators on $L_{p}(H)$ and that $L_{p}^{a}(H)$, whether defined by using $J_{1}^{\alpha}$ or $J_{2}^{\alpha}$, is equivalent to $D\left(T^{\alpha}\right)$ with the graph norm. We shall use Komatsu's definition and theory [4] of the powers $T^{\alpha}$.

Our comparative study of the Bessel potentials $J_{k}^{\alpha}$ will require no information about the operator $T$ beyond the facts that $(-T)$ and $\left(-T^{2}\right)$ generate bounded strongly continuous semi-groups $P_{z}$ and $H_{t}$ on a reflexive Banach space $X$. In Section 4 , where we study $J^{i \gamma}$, we need only the basic definitions and introduction of [2]; Section 5 has the same prerequisites. For these reasons we shall not review the formal definitions and basic measure theory of the spaces $L_{p}(H)$ and $L_{p}^{\alpha}(H)$, see $[2,3]$. We shall concentrate instead on listing some of the results from the BalalkrishnanKomatsu theory of fractional powers of operators. In what follows $T$ denotes a closed, densely defined operator on a reflexive Banach space $X$ such that $P_{z}=\exp (-z T)$ is a bounded, strongly continuous semi-group on $X$.

Early work on the theory of fractional powers of operators is surveyed in [7]. Balakrishnan [1], defined fractional powers $T^{\alpha}, 0<\alpha<1$, for an operator $(-T)$ which generates a bounded semi-group. In [1] the semi-group generated by $\left(-T^{\alpha}\right)$ is studied, formulas for the resolvent in terms of $\exp (-z T)$ are given, and properties of $T$ which are inherited by $T^{\alpha}$ are listed. Komatsu [4-I, II, III, IV] has developed an extensive theory of fractional powers of operators. In [4-I, II] it is assumed that $A$ is a linear operator (not necessarily densely defined) such that the negative half line is in the resolvent set of $A$ and $\left\|t(t+A)^{-1}\right\| \leqslant M$ for all $t>0 . A^{\alpha}$ is defined for all complex $a$ in Section 4 of [4-I]. For our purposes it will be sufficient to recall some of Komatsu's results for the case when $(-A)$ generates a bounded, strongly continuous semigroup on a reflexive Banach space $X$.

K-1. If $0<\operatorname{Re}(\alpha)<1, A^{\alpha} x=\frac{\sin \pi \alpha}{\pi} \int_{0}^{\infty} t^{\alpha-1} A(t+A)^{-1} x d t$ when $x \in D(A)$, the domain of $A ;$ [4-I, p. 299].

$\mathrm{K}-2$. If $0<\operatorname{Re}(\alpha)<\sigma<n, n$ a positive integer, then

$$
A^{\alpha} x=\frac{\Gamma(m)}{\Gamma(\alpha) \Gamma(m-\alpha)} \int_{0}^{\infty} t^{\alpha-1}\left(A(t+A)^{-1}\right)^{m} x d t
$$

for $x \in D\left(A^{N}\right)$ when $N>m>n$; [4-II, P. 92].

K-3. If $(-A)$ generates a bounded strongly continuous semi-group $T_{t}$ on $X$, then if $x \in D(A)$ and $0<\operatorname{Re}(\alpha)<\sigma<1$,

$$
A^{\alpha} x=\Gamma(-\alpha)^{-1} \int_{0}^{\infty}\left(T_{t} x-x\right) t^{-\alpha-1} d t ; \quad[4-\mathrm{I}, \text { p. 325]. }
$$

More formally, K-1 and K-3 define an operator $A_{\sigma}^{a}$ on a subspace $D^{\sigma}$ of $X ; D^{\sigma}$ is defined in [4-I]. If $A_{+}^{\alpha}$ denotes the smallest closed extension of $A_{\sigma}^{a}$, whose existence is proved in [4-I, Prop. 4.1], then $A^{\alpha}=A_{+}^{\alpha}$. Similarly K-2 defines an operator on a natural subspace of $X$ and its smallest closed extension is $A_{+}^{\alpha}=A^{\alpha}$ as is shown in [4-II]. When $\operatorname{Re}(\alpha)$ $<0, A_{-\sigma}^{\alpha}$ is defined by equation 4.10 of [4-I, p. 304] and $A_{-\sigma}^{\alpha .}$ is shown to have a smallest closed extension $A_{-}^{\alpha}$ which is independent of $\sigma$. When $\operatorname{Re}(\alpha)=0, A^{\alpha} x$ is defined by equation 4.11 of [4-I, p. 305] for $x \in D^{\sigma} \cap R^{\tau}$. There is the important

K-4. For every complex $\alpha, A_{\sigma \tau}^{\alpha}$ has the smallest closed extension $A_{0}^{\alpha}$ which is independent of $\sigma$ and $\tau$ when $-\tau<\operatorname{Re}(\alpha)<\sigma$. If $\operatorname{Re}(\alpha)>0$, $A_{0}^{\alpha}=A_{+}^{\alpha}$ on $D\left(A_{+}^{\alpha}\right) \cap \overline{R(A)}$ and if $\operatorname{Re}(\alpha)<0, A_{0}^{a}=A_{-}^{\alpha}$.

If $A$ has a bounded inverse, $R^{\sigma}=X$ and $A_{-}^{\alpha}$ is everywhere defined and analytic if $\operatorname{Re}(\alpha)<0$. If $x \in D^{\sigma}, A^{\alpha} x$ is analytic in $\operatorname{Re}(\alpha)<\sigma$. If $-(n+1)$ $<\operatorname{Re}(\alpha)<0$

$$
A_{-}^{\alpha}=\frac{-\sin \pi \alpha}{\pi} \frac{n !}{(\alpha+1) \ldots(\alpha+n)} \int_{0}^{\infty} t^{\alpha+n}(t+A)^{-n-1} d t
$$


and

K-5. If $\operatorname{Re}(\alpha)>0$, then $A_{+}^{\alpha}=A_{0}^{\alpha}$ is the inverse of $A_{0}^{-\alpha}=A_{-}^{-\alpha}$; the $D\left(A_{+}^{a}\right)$ is contained in the $R\left(A_{-}^{-\alpha}\right)$. See Section 5 of [4-I].

K-6. (i) If $\operatorname{Re}(\alpha) \cdot \operatorname{Re}(\beta)>0$, then $A_{\mp}^{a} A_{\mp}^{\beta}=A_{0}^{\alpha} A_{\mp}^{\beta}=A_{\mp}^{a+\beta}$ in the sense of the product of operators.

(ii) If $\alpha$ and $\beta$ are any complex numbers, then $\left[A_{0}^{\alpha} A_{0}^{\beta}\right]_{C}=A_{0}^{\alpha+\beta}$, where $[T]_{C}$ denotes the smallest closed extension of $T$.

(iii) If $A$ has a bounded inverse and if $\operatorname{Re}(\alpha)>0$, then $A_{0}^{\alpha} A_{0}^{\beta}$ $=A_{0}^{\alpha+\beta}$. See Section 7 of [4-I].

From the assumption that $\left\|t(t+A)^{-1}\right\| \leqslant M$ for $t>0$ and the resolvent equation it follows that $(t+A)^{-1}$ exists for $t$ in the sector $|\arg (t)|$ $<\operatorname{Arcsin}\left(M^{-1}\right)$ and that $t(t+A)^{-1}$ is bounded on each ray of this sector. Let $M(\theta)=\sup \left\{\left\|t(t+A)^{-1}\right\|:|\arg (t)|=\theta\right\}, \theta>0 ; M(\theta)$ is an increasing function of $\theta$. An operator $A$ is said to be of type $(\omega, M(\theta)), 0 \leqslant \omega<\pi$, if $A$ is closed, densely defined, the resolvent set of $(-A)$ contains the sector $|\arg (t)|<\pi-\omega$, and $\sup \left\{\left\|t(t+A)^{-1}\right\|:|\arg (t)|=\theta\right\} \leqslant M(\theta)<\infty$ holds for all $0 \leqslant \theta<\pi-\omega$. An operator $A$ is of type $(\omega, M(\theta))$ for an $\omega<\pi / 2$ if and only if $(-A)$ generates a semi-group $T_{t}$ which has an analytic extension to the sector $|\arg (t)|<\pi / 2-\omega$ such that the extension is uniformly bounded on each sector $|\arg (t)| \leqslant \pi / 2-\omega-\varepsilon$, for $\varepsilon>0$.

K-7. If $A$ is an operator of type $(\omega, M(\theta))$ and $0<\alpha \omega<\pi / 2$, then $\left(-A_{+}^{a}\right)$ is the generator of a strongly continuous semi-group $\exp \left(-t A_{+}^{\alpha}\right)$ which is analytic in the sector $|\arg (t)|<\pi / 2-\alpha \omega$ and uniformly bounded on each smaller sector $|\arg (t)|<\pi / 2-\alpha \omega-\varepsilon$, for $\varepsilon>0$. See Section 10 of [4-I].

K-8. Let $A$ be of type $(\omega, M(\theta))$. Then $\left(A_{+}^{\alpha}\right)^{\beta}=A_{+}^{\alpha \beta}$ if $0<\alpha<\pi / \omega$ and $\operatorname{Re}(\beta)>0$.

K-9. If $0<\alpha<1$ and if $T_{t}=\exp (-t A), \quad T_{t}^{\alpha} x=\exp \left(-t A_{+}^{\alpha}\right) x$ $=\int_{0}^{\infty} T_{s} x N(\alpha, t, s) d s$, where $N(\alpha, t, s)=(2 \pi i)^{-1} \int_{\sigma-i \infty}^{\sigma+i \infty} \exp \left(u s-t u^{\alpha}\right) d u ;$ [7].

Let $P_{z}=\exp (-z T)$ be the Poisson integral defined in the introduction; $P_{z}$ and $T$ have the following properties:

P-1. $P_{z}$ is a strongly continuous, contraction semi-group on $L_{p}(H)$; [2].

P-2. $P_{z}$ admits an extension as an analytic semi-group the sector $|\arg (z)|$ $<\pi / 4 ; P_{z}$ is uniformly bounded in each smaller sector.

This follows from the fact that $N_{t}(z)$ is analytic in $\operatorname{Re}(z)>0$ and the fact that the integral $P_{z}(f)=\int_{0}^{\infty} H_{t}(f) N_{t}(z) d t / t$ converges uniformly in compacts of $|\arg (z)|<\pi / 4-\varepsilon$, for $\varepsilon>0$.

P-3. If $H_{t} f=\int_{H} T_{y} f d n_{t} \circ B^{-1}(y)=\exp (-t A) f$, then $T=(A)^{1 / 2} ;[7$, p. 264].
P-4. If $B$ is a one-one Hilbert-Schmidt operator, $T$ is one-one on $L_{p}(H)$ and the range of $T, R(T)$, is dense in $L_{p}(H)$.

If $T$ is one-one on $L_{p}(H)$, the $R(T)$ is dense in $L_{p}(H)$ by Theorem 3.1 of $[4-\mathrm{I}]$. To show that $T$ is one-one, it suffices to show that $T^{2}$ is one-one. If $T^{2} f=0, H_{t} f=f$ for all $t>0$. If $A_{h}$ denotes the infinitesmal generator of $T_{t B h}, t>0$, then $A_{h} H_{t^{2}}(f)=A_{h} f$ for all $h$ in $H$. From the formula for $A_{h} H_{t^{2}}$ given in [2], it can be seen that $Z_{\delta_{e}}(f)=\int_{\delta}^{e} A_{h} H_{t^{2}}(f) d t=$ $=A_{h} f(\varrho-\delta)$ is a bounded operator on $L_{p}(H)$ with norm at most $K \log (\varrho ! \delta)$. After dividing by $(\varrho-\delta)$, one shows that this inequality implies that $A_{h} f=0$ for all $h$ in $H$ by letting $\varrho \rightarrow \infty$. $A_{h} f=0$ for all $h$ in $H$ implies that $T_{t B h} f=f$ for all $t>0$. A well-known result due to Hormander implies that for tame functions $g$, $\left\|T_{t B h} g+g\right\|_{p} \rightarrow 2^{1 / p}\|g\|_{p}$ as $t$ tends to $+\infty$. Since the tame functions in $L_{p}(H)$ are dense in $L_{p}(H)$, this limit holds for all $f$ in $L_{p}(H)$. For the $f$ with $T^{2} f=0,2\|f\|_{p}=\left\|T_{t B h} f+f\right\|_{p}$ $\rightarrow 2^{1 / p}\|f\|_{p}$. This implies that $f=0$ and $T$ is one-one.

2. Basic properties of Bessel potentials. In Section 3 of [3] we studied the Bessel potential $J^{\alpha}$ which is mentioned in the introduction of the present paper. We showed that for $\operatorname{Re}(\alpha)>0, J^{\alpha}$ is bounded on $L_{p}(H)$, $J^{\alpha}$ is strongly analytic, $\lim \left\{J^{\alpha} f:|\arg (\alpha)| \leqslant \theta<\pi / 2, \alpha \rightarrow 0\right\}=f, J^{\alpha} J^{\beta}$ $=J^{\alpha+\beta}, J^{\alpha}$ is the $\alpha$-th Komatsu power of $J^{\prime}, J^{\alpha}$ is one-one on $L_{p}(H)$, $R\left(J^{\alpha}\right)$ is dense in $L_{p}(H), T^{\alpha} J^{\alpha}$ is bounded on $L_{p}(H)$, and $\left(-A_{h}\right)^{\alpha} J^{a}$ is bounded on $L_{p}(\dot{H})$ when $A_{h}$ is the infinitesmal generator of the translation semi-group $T_{t B h}, t>0$, and $B$ is the one-one Hilbert-Schmidt operator of Section 1. In Sections 2 and 3 of the present paper we shall study abstract Bessel potentials by using the theory of fractional powers of operators.

Let $T$ be a one-one, closed, densely defined operator on a reflexive Banach space $X$ such that $(-T)$ and $\left(-T^{2}\right)$ generate strongly continuous, contraction semi-groups $P_{z}=\exp (-z T)$ and $H_{t}=\exp \left(-t T^{2}\right)$. Then $P_{z}$ can be written as an integral of $H_{t}$ as in Section 1 and $P_{z}$ is analytic in $|\arg (z)|<\pi / 4$. Let $J_{1}^{\alpha}$ and $J_{2}^{\alpha}$ be as in Section 1 when $\operatorname{Re}(\alpha)>0$. Recall that since $X$ is reflective, it $T_{t}=\exp (-t A)$ is a bounded strongly continuous semi-group, then $X=N(A) \otimes \overline{R(A)}$ by Theorem 3.1 of [4-I]. Since $T$ is one-one, $R(T)$ and $R\left(T^{2}\right)$ are dense in $X$. We begin by listing some of the basic properties of the $J_{k}^{\alpha}$.

THEOREM 1. Let $\alpha$ and $\beta$ be positive real numbers. Then for $k=1,2$ :

(1) $\left\|J_{k}^{\alpha}\right\| \leqslant 1$.

(2) $\lim \left\{J_{k}^{a} f: \alpha \rightarrow 0\right\}=f$.

(3) $J_{k}^{\beta}=\left(J_{k}^{1}\right)^{\beta}$, the $\beta$-th power of $J_{k}^{1}$.

(4) $J_{k}^{a}=\left(1+T^{k}\right)^{-a / k}$. 
(5) $R\left(J_{k}^{\alpha}\right)$ is dense in $L_{p}(H)$.

(6) $J_{k}^{\alpha} J_{k}^{\beta}=J_{k}^{\alpha+\beta}$.

(7) $J_{k}^{a}$ is one-one on $L_{p}(H)$.

Proof. Let $T_{t}$ denote a contraction semi-group, $T_{t}=\exp (-t A)$, which stands for $H_{t}$ or $P_{y}$ in the proof. Set $J^{\beta} f=\Gamma(\beta)^{-1} \int_{0}^{\infty} T_{t} f t^{\beta-1} e^{-t} d t$. (1) follows from Minkowski's integral inequality. For (2) let $f \in X$, $\varepsilon>0$, and $\delta>0$ such that if $0<t<\delta,\left\|T_{t} f-f\right\|<\varepsilon$. Choose $\eta>0$ such that if $0<\beta<\eta, \Gamma(\beta)^{-1} \int_{\delta}^{\infty} t^{\beta-1} e^{-t} d t<\varepsilon$. Then if $\beta<\eta,\left\|J^{\beta} f-f\right\|$ $\leqslant\left\|\Gamma(\beta)^{-1} \int_{0}^{\delta}\left(T_{t} f-f\right) t^{\beta-1} e^{-t} d t\right\|+\left\|\Gamma(\beta)^{-1} \int_{\delta}^{\infty}\left(T_{t} f-f\right) t^{\beta-1} e^{-t} d t\right\| \leqslant \varepsilon+2 \varepsilon\|f\|$. This proves (2). To prove (3) and (4) let $\beta>0$ and set $J f=J^{1} f=\int_{0}^{\infty} T_{t} f e^{-t} d t$ $=(1+A)^{-1} f$; we will show that $(1+A)^{-\beta} f=J^{\beta} f$. Let $0<\beta<1$; then for $x$ in $X$

$$
\begin{aligned}
J^{\beta} x & =\Gamma(\beta)^{-1} \int_{0}^{\infty} u^{\beta-1} e^{-u} T_{u} x d u \\
& =\Gamma(\beta)^{-1} \Gamma(1-\beta)^{-1} \int_{0}^{\infty}\left(\int_{0}^{\infty} t^{-\beta} e^{-u} e^{-t u} d t\right) T_{u} x d u \\
& =\Gamma(\beta)^{-1} \Gamma(1-\beta)^{-1} \int_{0}^{\infty}\left(\int_{1}^{\infty}(t-1)^{-\beta} e^{-t u} d t\right) T_{u} x d u \\
& =\Gamma(\beta)^{-1} \Gamma(1-\beta)^{-1} \int_{1}^{\infty}(t-1)^{-\beta} \int_{0}^{\infty} e^{-t u} T_{u} x d u d t \\
& =\Gamma(\beta)^{-1} \Gamma(1-\beta)^{-1} \int_{0}^{\infty}(t-1)^{-\beta}(t+A)^{-1} x d t \\
& =\Gamma(\beta)^{-1} \Gamma(1-\beta)^{-1} \int_{0}^{\infty} v^{-\beta}(v+1+A)^{-1} x d v .
\end{aligned}
$$

Since $(v+1+A)^{-1}=J(v J+1)^{-1}$, set $w=v^{-1}$ to get

$$
J^{\beta} x=\frac{\sin \pi \beta}{\pi} \int_{0}^{\infty} w^{\beta-1} J(w+J)^{-1} x d w
$$

by K-1. This completes the proof of (3) and (4) for $0<\beta<1$; the general result now follows from K-6. (5) follows from K-5 since $J_{k}^{a}=\left(1+T^{/ k}\right)^{\text {-alk }}$ $=\left[\left(1+T^{k}\right)^{a / k}\right]^{-1}$ and $D\left(\left(1+T^{k /}\right)^{a / k}\right) \subset R\left(J_{k}^{a}\right) .(6)$ is a consequence of $(4)$ and $\mathrm{K}-6$; an elementary direct computation also verifies the desired identity. If $J_{k}^{\alpha}(f)=0, J_{k}^{a+r}(f)=0$ for $r>0$ by $(6)$. Since $J_{t_{c}}^{\alpha}(f)$ extends to an analytic function in $\operatorname{Re}(\alpha)>0$, the uniqueness principle for analytio functions implies that $J_{k}^{\alpha}(f)=0$ for all $\operatorname{Re}(\alpha)>0$. By $(1), . f=0$. So (7) holds and $J_{k}^{a}$ is one-one.

Let $T^{\alpha}, \alpha>0$, be as in K-1, K-2, or K-3; we shall examine $T^{\alpha} J_{k}^{\alpha}$.

THEOREM 2. $T^{\alpha} J_{k}^{\alpha}$ is a bounded operator on $X$ for $k=1,2$ if $\alpha>0$. These operators are given by:

$$
T^{\alpha} J_{1}^{\alpha} x=x-\frac{\sin \pi \alpha}{\pi} \int_{0}^{1} J_{u} x u^{\alpha}(1-u)^{-\alpha} d u \quad \text { for } \quad 0<\alpha<1
$$

and

$$
T^{\alpha} J_{2}^{\alpha} x=x-\frac{\sin (\pi \alpha / 2)}{\pi} \int_{0}^{1} K_{u} x u^{\alpha / 2}(1-u)^{-\alpha / 2} d u
$$

for $0<\alpha<2$, where $J_{u} x=\int_{0}^{\infty} e^{-u t} P_{t} x d t$ and $K_{u} x=\int_{0}^{\infty} e^{-u t} H_{t} x d t$.

Proof. To prove the theorem for $J_{k}^{a}, k=1,2$ note first that $T(1+T)^{-1}$ $=1-(1+T)^{-1}$ and $T^{2}\left(1+T^{2}\right)^{-1}=1-\left(1+T^{2}\right)^{-1}$ are bounded operators, so that we need only prove the theorem for $0<\alpha<k$ for $J_{k}^{\alpha}$; K-6 can be used to complete the proof of the boundedness of $T^{\alpha} J_{k}^{\alpha}$. Let $T_{t}=\exp (-t A)$ be a bounded, strongly continuous semi-group on $X$. We shall show that

$$
A^{\beta}(1+A)^{-\beta} x=x-B(\beta, 1-\beta)^{-1} \int_{0}^{1}(u+A)^{-1} x u^{\beta}(1-u)^{-\beta} d u
$$

for $0<\beta<1$. Here $B(x, y)$ is the $\beta$-function.

For $0<\beta<1, J^{\beta} x=\Gamma(\beta)^{-1} \int_{0}^{\infty} T_{t} x t^{\beta-1} e^{-t} d t=(1+A)^{-\beta} x$ and $A^{\beta} x$ $=\Gamma(-\beta)^{-1} \int_{0}^{\infty}\left(T_{y} x-x\right) y^{-1-\beta} d y$. If $x \in D(A)$,

$$
A^{\beta} x=-\Gamma(1-\beta)^{-1} \int_{0}^{\infty} T_{y}^{\prime} x y^{-\beta} d y
$$

where $T_{y}^{\prime} x=\frac{\partial}{\partial y} T_{y} x$. Let $L g(u)$ denote the Laplace transform of $g$ at $u$. Then

$$
A^{\beta} J^{\beta} x=-\frac{\sin \pi \beta}{\pi} \int_{1}^{\infty} L\left(t^{\beta} A_{0}^{\beta} T_{t} x\right)(u) d u,
$$

where $A_{0}^{\beta}=\Gamma(-\beta) A^{\beta}$. Then

$$
t^{\beta} A_{0}^{\beta}\left(T_{t} x\right)=t^{\beta} \int_{0^{+}}^{\infty} \frac{\partial}{\partial y} T_{y+t} x y^{-\beta} d y=\int_{0^{+}}^{\infty} \frac{\partial}{\partial t} T_{y+t} x t^{\beta} y^{-\beta} d y=\int_{0^{+}}^{\infty} t T_{t}^{\prime} T_{t y} x y^{-\beta} d y .
$$


But $T_{t}^{\prime} T_{t y} x=(y+1)^{-1} \frac{\partial}{\partial t} T_{t(y+1)} x$. Then

$$
\begin{aligned}
L\left(t^{\beta} A_{0}^{\beta} T_{t} x\right)(u) & =\int_{0^{+}}^{\infty}(y+1)^{-1}\left(-\frac{\partial}{\partial u} u\right) L\left(T_{t(y+1)} x\right)(u) y^{-\beta} d y \\
& =\int_{0^{+}}^{\infty}(y+1)^{-2}\left(-\frac{\partial}{\partial u} u\right) J_{u(y+1)-1}(x) y^{-\beta} d y
\end{aligned}
$$

where $J_{v} x=\int_{0^{+}}^{\infty} e^{-v t} T_{t} x d t$. Since $\left\|\left(-\frac{\partial}{\partial u} u\right) J_{u(y+1)^{-1}(x)}\right\| \leqslant K(y+1) u^{-1}\|x\|$, we consider an interchange of integrals in $\int_{i}^{R} L\left(t^{\beta} A_{0}^{\beta} T_{t}(x)\right)(u) d u$.

$$
\int_{1}^{R}(1+y)^{-2}\left(-\frac{\partial}{\partial u} u\right) J_{u(1+y)-1}(x) d u=(y+1)^{-2}\left[J_{(1+y)}-1-R J_{R(1+y)}-1\right](x) .
$$

Since $\left\|R J_{R(y+1)-1}(x)\right\| \leqslant K(y+1)\|x\|$, the dominated convergence theorem implies that

$$
\begin{aligned}
A^{\beta} J^{\beta} x & =-\frac{\sin \pi \beta}{\pi} \int_{0^{+}}^{\infty}\left(J_{(1+y)^{-1}}(x)-(y+1) x\right) y^{-\beta}(1+y)^{-2} d y \\
& =-\frac{\sin \pi \beta}{\pi} \int_{0^{+}}^{1}\left(J_{u} x-u^{-1} x\right) u^{\beta}(1-u)^{-\beta} d u \\
& =x-\frac{\sin \pi \beta}{\pi} \int_{0}^{1} J_{u} x u^{\beta}(1-u)^{-\beta} d u .
\end{aligned}
$$

Since $\left\|J_{u} x\right\| \leqslant K u^{-1}\|x\|,\left\|A^{\beta} J^{\beta} x\right\| \leqslant(1+K)\|x\|$ by the triangle inequality for integrals. This completes the proof of Theorem 2.

Remark 2.1. When $\boldsymbol{P}_{t}$ and $H_{t}$ are semi-groups on $L_{p}(H)$ of the type given in the introduction, $T^{\alpha} J_{k}^{\alpha}$ is given by convolution with a measure on $H$ for all $\alpha>0$; see [3]. If $X=L_{p}$ of an Abelian group and if $P_{t}$ and $H_{t}$ are given by convolution with measures, it is easy to see from Theorem 2 above or from Theorem 4 of [3], that $T^{\alpha} J_{k}^{\alpha}$ is given by convolution with a measure.

Remark 2.2. Many of the properties of the $J_{k}^{\alpha}$ in Theorem 1 also hold for complex $\alpha$ when $\operatorname{Re}(\alpha)>0$. Theorem 2 holds for these complex $\alpha$ with no change in proof or notation.

Remark 2.3. For any $k$ in $0<k \leqslant 2, J_{k}^{a}=\left(1+T^{k}\right)^{-a / k}$ defines a strongly analytic semi-group in $\alpha$ when our present assumptions on $P_{z}=\exp (-z T)$ are in force. It will not be hard to see in what follows that any of the $J_{k}^{a}, k_{1}=k_{1}, k_{2}$ are equivalent for $0<k_{1}, k_{2} \leqslant 2$. The basis for this assertion is the set of results presented in Section 10 of [4-I] regarding the semi-groups generated by fractional powers of operators.

3. Equivalence of $J_{1}^{\alpha}$ and $J_{2}^{\alpha}$. By statement (7) of Theorem $1, J_{k}^{\alpha}$ is one-one on $X$ for $k=1,2$. Define $X_{k}^{\alpha}=R\left(J_{k}^{\alpha}\right)$ with the norm $\|y\|_{\alpha, ~}$ $=\|x\|$ if $J_{k}^{\alpha} x=y$. In this section we shall complete the proof of the equivalence of the norms $\|_{\alpha, k}$ by proving that $\left(J_{k}^{a}\right)^{-1} J_{i}^{a}$ is a bounded operator on $X$ for $k, i=1,2$ and $\alpha>0$; this leads to the conclusion that $X^{\alpha}$ is equivalent to $D\left(T^{\alpha}\right)$ when this domain is equipped with the graph norm. The following lemma will be useful.

LEMMA 3.1. Let $(-A)$ be the infinitesmal generator of a bounded, strongly continuous semi-group on $X$ and let $0<\alpha<1$. If $x \in D(A),(1+A)^{a}$ $x=A^{a} x+B x$, where $B$ is a bounded operator on $X$

Proof. By K-1

$$
\begin{aligned}
(1+A)^{\alpha} x & =\frac{\sin \pi \alpha}{\pi} \int_{0}^{\infty} t^{\alpha-1}(A+1)(t+1+A)^{-1} x d t \\
& =\frac{\sin \pi \alpha}{\pi} \int_{0}^{\infty} t^{\alpha-1}(t+1+A)^{-1} x d t+\frac{\sin \pi \alpha}{\pi} \int_{0}^{\infty} t^{\alpha-1} A(t+1+A)^{-1} x d t .
\end{aligned}
$$

Since $\left\|(t+1+A)^{-1}\right\| \leqslant K(t+1)^{-1}$, the first integral on the right represents a bounded operator on $X$. By the resolvent equation, $(t+1+A)^{-1}-(t+A)^{-1}$ $=-(t+1+A)^{-1}(t+A)^{-1}$. Then

$$
\begin{aligned}
\frac{\sin \pi \alpha}{\pi} \int_{0}^{\infty} t^{\alpha-1} A(t+1+A)^{-1} x d t & \\
& =A^{\alpha} x-\frac{\sin \pi \alpha}{\pi} \int_{0}^{\infty} t^{\alpha-1} A(t+1+A)^{-1}(t+A)^{-1} x d t .
\end{aligned}
$$

Since $\left\|A(t+A)^{-1}\right\| \leqslant K$ and $\left\|(t+1+A)^{-1}\right\| \leqslant K(t+1)^{-1}$, the last integral on the right represents a bounded operator on $X$. Thus $(1+A)^{\alpha}$ has the desired form.

THEOREM 3. If $\alpha>0,\left(J_{k}^{\alpha}\right)^{-1} J_{i}^{\alpha}$ are bounded operators on $X$ if $i, k=1,2$.

Proof. Consider $\left(1+T^{2}\right)^{\alpha / 2}(1+T)^{-\alpha}$ and write $\alpha=2 n+\beta$, where $0 \leqslant \beta<2$. Then by Lemma 3.1, $\left(1+T^{2}\right)^{\beta / 2}=\left(T^{2}\right)^{\beta / 2}+B$, where $B$ is a bounded operator. By K-8, $\left(T^{2}\right)^{\beta / 2}=T^{\beta}$. Since $\left(1+T^{2}\right)^{\alpha / 2}=\left(1+T^{2}\right)^{n}$ $\left(T^{\beta}+B\right)=\sum_{k=0}^{n} A_{k} T^{2 k}+\sum_{k=0}^{n} B_{k} T^{2 k+\beta}$, where $A_{k}$ and $B_{k}$ are bounded operators on $X$, and since $T^{\gamma}(1+T)^{-a}=T^{\gamma}(1+T)^{-\gamma}(1+T)^{-(\alpha-\gamma)}$ for $\gamma \leqslant \alpha$, Theorem 2 implies that $\left(1+T^{2}\right)^{\alpha / 2}(1+T)^{-\alpha}$ is a bounded operator on $X$. 
Similarly, write $\alpha=N+\gamma$, where $N$ is a non-negative integer and $0 \leqslant \gamma<1$. Then $(1+T)^{\alpha}=(1+T)^{N}\left(T^{\gamma}+B\right)=\sum_{k=0}^{N} A_{k} T^{k}+\sum_{k=0}^{N} B_{k} T^{k+\gamma}$, where $A_{k}$ and $B_{k}$ are bounded operators on $X$. Since if $\delta \leqslant \alpha, T^{\delta}\left(1+T^{2}\right)^{-\alpha / 2}$ $=\left(T^{2}\right)^{\delta / 2}\left(1+T^{2}\right)^{-\delta / 2}\left(1+T^{2}\right)^{-(\alpha-\delta) / 2}$ by $K-8$ and Theorem 2 implies that $(1+T)^{\alpha}\left(1+T^{2}\right)^{-\alpha / 2}$ is a bounded operator on $X$. This completes the proof of Theorem 3 .

Our methods give the following

CoRollary 3.1. $X_{k}^{a}, k=1,2$, is equivalent to $D\left(T^{\alpha}\right)$ when $D\left(T^{\alpha}\right)$ is equipped with the graph norm.

Proof. By Theorem 3, it suffices to prove that $X_{1}^{\alpha}=D\left(T^{\alpha}\right)$. If $y=J_{1}^{a} x$, then $\|y\|+\left\|T^{\alpha} J_{1}^{\alpha} x\right\| \leqslant K(\alpha)\|x\|=K(\alpha)\|y\|_{\alpha, 1}$ by Theorem 2. $\underset{N}{\text { Write }}\|y\|_{\alpha, 1}=\|x\|=\left\|(1+T)^{\alpha} y\right\|$, and expand $(1+T)^{\alpha}=\sum_{k=0}^{N} A_{k} T^{k}+$ $+\sum^{N} B_{k} T^{k+\beta}$ by Lemma 3.1. By Theorem 6.5 of [4-I], $D\left(T^{\alpha}\right) \subset D\left(T^{\gamma}\right)$ continuously if $\gamma<\alpha$. Thus $\left\|(1+T)^{\alpha} y\right\| \leqslant K(\alpha)\left(\|y\|+\left\|T^{\alpha} y\right\|\right)$ and the proof is complete.

COROLLARY 3.2. $X_{i}^{a}$ consists precisely of those elements $x$ of $X$ for which $T^{\alpha} x$ is also in $X$.

Stein [6] has studied the questions dealt with in this section over finite dimensional Euclidean spaces. Fourier multiplier techniques are used in [6] to prove the above results.

4. BoundeDness of $J^{i \gamma}$. In this section we shall prove that $J^{i \gamma}$, $\gamma$ real, is a group of bounded operators on $L_{p}(H)$ if $1<p<\infty$, and we will study the relationship between $J^{i \gamma}$ and the closed operator $(1+A)^{-i \gamma}$. If $\gamma=0, J^{i \gamma}$ is the identity on $L_{p}(H)$ and if $\gamma \neq 0$, set

$$
J^{i \gamma} f=\left[\lim _{\varepsilon \rightarrow 0} \Gamma(i \gamma)^{-1} \int_{\varepsilon}^{\infty} P_{t} f t^{i \nu-1} e^{-t} d t+\Gamma(i \gamma+1)^{-1} \varepsilon^{i \gamma} f\right] .
$$

Here $P_{t} f$ is the Poisson integral of $f$ as defined and studied in [2], and which is briefly described in Section 1 of this paper. In [2] it was shown that there is a unique Borel probability measure $p(E)$ on $H$ such that if $p_{t}(E)=p(E / t)$ for $t>0, P_{t} f=\int_{I I} T_{y} f d p_{t}(y)=\int_{H} T_{t y} f d p(y)$ for all $f$ in $L_{p}(H) ; y \rightarrow T_{y}$ is the regular representation of the additive group of $H$ acting on $L_{p}(H)$.

For $\alpha=\beta+i \gamma, \beta \geqslant 0$ and $\gamma$ real, define $J_{\varepsilon}^{\alpha} f=f$ if $\alpha=0$ and if $\operatorname{Re} \alpha \neq 0$ define

$$
J_{\varepsilon}^{\alpha} f=\Gamma(\alpha)^{-1} \int_{\varepsilon}^{\infty} P_{t} f t^{\alpha-1} e^{-t} d t+\varepsilon^{\alpha} \Gamma(\alpha+1)^{-1} f \quad \text { for } \varepsilon>0 .
$$

Then for $\operatorname{Re} \alpha>0, J_{\varepsilon}^{\alpha}$ converges strongly to $J^{\alpha} f$ as $\varepsilon$ tends to zero. Define $J^{\alpha} f=\lim \left\{J_{\varepsilon}^{\alpha} f: \varepsilon \rightarrow 0^{+}\right\}$for $\operatorname{Re} \alpha \geqslant 0$ if this limit exists. Let $1<p<\infty$ and $1 / p+1 / q=1$.

THEOREM 4. $J^{i \gamma}$ is a bounded operator on $L_{p}(H)$ for all real $\gamma$ and $\left\|J^{i \gamma}\right\| \leqslant K p q(|\gamma|+1)^{2}|\Gamma(i \gamma+1)|^{-1}$.

Proof. First consider $\left(T_{\varepsilon}^{a} f\right)(x)=\int_{\varepsilon}^{\infty} f(x-t) \exp (-t / a) t^{i \gamma-1} d t$ on $L_{p}((-\infty, \infty), d x)$. Let $g(t)=t^{i \gamma-1}$ if $t>0$ and $g(t)=0$ if $t<0$. Then for $a>0$,

Since

$$
\left(T_{\varepsilon}^{a} f\right)(x)=\int_{|t|>\varepsilon} f(x-t) \exp (-|t| / a) g(t) d t
$$

$$
\exp (-|t| / a)=(\pi)^{-\mathrm{\tau}} \int_{-\infty}^{\infty} e^{-i t y} \frac{a d y}{1+y^{2} a^{2}}
$$

set $h(a, y)=a(\pi)^{-1}\left(1+y^{2} a^{2}\right)^{-1}$ and write

$$
\left(T_{\varepsilon}^{\alpha} f\right)(x)=\int_{-\infty}^{\infty} e^{-i x y} \int_{|t|>\varepsilon} f(x-t) e^{i(x-t) y} g(t) d t h(a, y) d y .
$$

By Minkowski's integral inequality and by Theorem 1 of [5],

$$
\left\|T_{\varepsilon}^{a}\right\|_{p} \leqslant K p q(|\gamma|+1)^{2}|\gamma|^{-1} \text { for all } \varepsilon>0 .
$$

We may write

$$
J_{\varepsilon}^{i \gamma} f=\Gamma(i \gamma)^{-1} \int_{H}\left[\int_{\varepsilon}^{\infty} T_{t y} f t^{i \gamma-1} e^{-t} d t+\frac{\varepsilon^{i \gamma}}{i \gamma} f\right] d p(y) .
$$

If $f$ is a bounded tame function on $H$, then the rotational invariance of the normal distribution can be used as in the proof of Proposition 3 of [2] to show that as a consequence of the above inequality for $T_{\varepsilon}^{\|y\|}$,

$$
\left\|\int_{\varepsilon}^{\infty} T_{t y} f t^{i \gamma-1} e^{-t} d t\right\| p \leqslant K(\gamma, p)\|f\|_{p}
$$

for all $\varepsilon>0$. The bounded tame functions are dense in $L_{p}(H)$. Minkowski's integral inequality can be used to complete the proof that $\left\|J_{\varepsilon}^{i \gamma} f\right\|_{p}$ $\leqslant K p q(|\gamma|+1)^{2}|\Gamma(i \gamma+1)|^{-1}$ for all $\varepsilon>0$.

To prove convergence as $\varepsilon \rightarrow 0^{+}$, write

$$
\begin{aligned}
J_{\varepsilon}^{i \gamma} f=\Gamma(i \gamma)^{-1} \int_{H .}\left[\int_{1}^{\infty} T_{t y} f t^{i \gamma-1} e^{-t} d t\right. & +\int_{\varepsilon}^{1} T_{t y} f t^{i \gamma-1}\left(e^{-t}-1\right) d \dot{t}+ \\
& \left.+\left(\int_{\varepsilon}^{1} T_{t y} f t^{i \gamma-1} d t+\frac{\varepsilon^{i \gamma} f}{i \gamma}\right)\right] d p(y) .
\end{aligned}
$$


The first and second integrals on the right converge absolutely. If $f$ is a bounded tame function with bounded derivatives, write the third quantity as

$$
\int_{\varepsilon}^{1} T_{t y} f t^{i \gamma-1} d t+\frac{\varepsilon^{i \gamma}}{i \gamma} f=\int_{\varepsilon}^{1}\left(T_{t y} f-f\right) t^{i \gamma-1} d t+\frac{f}{i \gamma} .
$$

Since $\left\|T_{t y} f-f\right\|_{p} \leqslant K t\|y\|$ and since $\int_{H}\|y\| d p(y)<\infty$, the third quantity on the right above converges as $\varepsilon \rightarrow 0^{+}$. Thus $J_{\varepsilon}^{i \gamma}$ converges strongly to a bounded operator $J^{i \gamma}$ and $\left\|J^{i \gamma}\right\| \leqslant K p q(|\gamma|+1)^{2}|\Gamma(i \gamma+1)|^{-1}$.

The next theorem shows that $\int_{0}^{\infty} P_{t} f t^{i \nu-1} d t$ converges with respect to a certain summability method.

TheOREM 5. $J^{i \gamma} f=\lim \left\{J^{\beta+i \gamma} f: \beta \rightarrow 0^{+}\right\}$for all $f$ in $L_{p}(H)$.

Proof. The integral $\Gamma(\alpha)^{-1} \int_{1}^{\infty} P_{t} f t^{\alpha-1} e^{-t} d t, \alpha=\beta+i \gamma$, converges uniformly to $\Gamma(i \gamma)^{-1} \int_{1}^{\infty} P_{t} f t^{i \gamma-1} e^{-t} \frac{1}{d t}$ as $\beta \rightarrow 0^{+}$. It is sufficient to consider the limit of $\int_{0}^{I} P_{t} f t^{\alpha-1} e^{-t} d t$. This last integral is

$$
\int_{0}^{1} \beta \dot{x}^{\beta-1} \int_{x}^{1} P_{t} f t^{i \gamma-1} e^{-t} d t d x .
$$

The function $\beta x^{\beta-1}$ gives a regular summability method on $0 \leqslant x \leqslant 1$. Since the integral $\int_{0^{+}}^{1} P_{t} f t^{\alpha-1}\left(e^{-t}-1\right) d t$ converges strongly to $\int_{0+}^{1} P_{t} f t^{i \gamma-1}\left(e^{-t}-1\right) d t$ as $\beta \stackrel{0^{+}}{\rightarrow} 0^{+}$, we consider

$$
\lim _{\beta \rightarrow \infty} \int_{0}^{1} \beta x^{\beta-1} \int_{x}^{1} P_{t} f t^{i \gamma-1} d t d x .
$$

From Section 3 of [5], we have that this last limit exists if

$$
\lim _{\varepsilon \rightarrow \infty}\left[\int_{\varepsilon}^{1} P_{t} f t^{i \gamma-1} d t+\frac{\varepsilon^{i \gamma}}{i \gamma} f\right]
$$

exists; when the last limit exists these limits are equal. Theorem 4 shows that the last limit exists, so that

$$
J^{i \gamma} f=\lim \left\{{ }^{\beta+i \gamma} f: \beta \rightarrow 0^{+}\right\} .
$$

CoRollary 4.1. If $\alpha$ is a real positive number, $J^{\alpha} J^{i \gamma}=J^{\alpha+i \gamma}$.

Proof: For $0<\varepsilon<a, \quad\left\|J^{\alpha} J^{i \gamma} f-J^{a+i \gamma} f\right\|_{p} \leqslant\left\|J^{s} J^{i \gamma} f-J^{s+i \gamma} f\right\|_{p}$ by statements 6 and 1 in Theorem 1 . By the triangle inequality $\| J^{z} J^{i \gamma} f-$ $-J^{s+i \gamma} f\left\|_{p} \leqslant\right\| J^{z} J^{i \gamma} f-J^{i \gamma} f\left\|_{p}+\right\| J^{i \nu} f-J^{s+i \gamma} f \|_{p}$. By statement 2 of Theorem 1 and by Theorem 5 , the terms on the right tend to 0 as $\varepsilon \rightarrow 0^{+}$. This verifies the required identity.
COROLLARY 4.2. $T^{a}=J^{\alpha+i \gamma}, \alpha \geqslant 0$, forms a bounded, strongly continuous family of operators on $L_{p}(H)$ with $\left\|T^{\alpha}\right\| \leqslant K p q(|\gamma|+1)^{2}|\Gamma(i \gamma+1)|^{-1}$.

Proof. Since $T^{\alpha}=J^{\alpha} J^{i \gamma}$ and $J^{\alpha}$ is strongly continuous for $a>0$ and $J^{i \gamma}$ is bounded, $T^{\alpha}$ is strongly continuous. The estimate for $\left\|T^{\alpha}\right\|$ follows from the estimate for $J^{a},\left\|J^{a}\right\| \leqslant 1$. and the estimate for $\left\|J^{i \gamma}\right\|$ which is given in Theorem 4.

COROLLARY 4.3. $J^{i \gamma} J^{i \beta}=J^{i(\gamma+\beta)}$ for all real $\gamma$ and $\beta$.

Proof. If $\varepsilon>0, J^{2 \varepsilon+i \gamma} J^{i \beta}=J^{i \gamma} J^{2 \varepsilon+i \beta}=J^{2 \varepsilon+i(\gamma+\beta)}$ by part 6 of Theorem 1 and Corollary 4.1. By Theorem 5, if we take the limit as $\varepsilon \rightarrow 0^{+}$ in this equation, we get the desired result.

COROLLARY 4.4. $J^{i \gamma}, \gamma$ real, is a strongly continuous group of operators on $L_{p}(H)$ with $J^{i 0}=$ the identity and $\left(J^{i \gamma}\right)^{-1}=J^{-i \gamma}$.

Proof. Because of Corollary 4.3 we need only show that $\lim \left\{J^{i \gamma} f\right.$ : $\gamma \rightarrow 0\}=f$ for each $f$ in $L_{p}(H)$. The bound on $\left\|J^{J^{i \nu}}\right\|$ is

$$
K p q(|\gamma|+1)^{2}|\Gamma(i \gamma+1)|^{-1}=K p q(\pi|\gamma|)^{-1 / 2}(\sinh \pi|\gamma|)^{+1 / 2}(|\gamma|+1)^{2},
$$

since $|\Gamma(i \gamma)|=(\pi)^{1 / 2}(\gamma \sinh \pi \gamma)^{-1 / 2}$ as follows from the well-known identity for $\Gamma(z) \Gamma(1-z)$. Thus $\left\|J^{i \gamma}\right\|$ is bounded on any compact neighborhood of $\gamma=0$ and $\lim \left\{J^{\varepsilon+i \gamma} f: \varepsilon \rightarrow 0^{+}\right\}=J^{i \gamma} f$ uniformly on $-1 \leqslant \gamma \leqslant 1$. Because of the strong continuity of $J^{\alpha}$ in $\operatorname{Re} \alpha>0$, the following equality completes the proof:

$$
\lim _{\gamma \rightarrow 0} J^{i \gamma} f=\lim _{\gamma \rightarrow 0} \lim _{\varepsilon \rightarrow 0} J^{\varepsilon+i \gamma} f=\lim _{\varepsilon \rightarrow 0} \lim _{\gamma \rightarrow 0} J^{\varepsilon+i \gamma} f=\lim _{\varepsilon \rightarrow 0} J^{\varepsilon} f=f .
$$

COROLLARY 4.5. $J^{i \gamma} f=(1+T)^{-i \gamma} f=\left[(1+T)^{i \gamma}\right]^{-1} f$, for all $f$ in $L_{p}(H)$, and $(1+T)^{-i \gamma}$ is a bounded operator on $L_{p}(H)$ for all real $\gamma$.

Proof. By Corollary 5.3 of [4-I], $J^{\alpha+i \gamma}$ is the inverse of $(1+T)^{\alpha+i \gamma}$ if $\alpha>0$. Because the fractional powers are strongly continuous on a dense set of $L_{p}(H)$ in a strip $-\tau<\alpha<\sigma,-\infty<\gamma<\infty$, by Theorem 8.2 of [4-I], we have that $J^{i \gamma} f=(1+T)^{-i \gamma} f=\left[(1+T)^{i \gamma}\right]^{-1} f$ for a dense set of $f$ 's in $L_{p}(H)$. Since the $J^{\alpha+i \gamma}=\left((1+T)^{-1}\right)^{\alpha+i \gamma}=(1+T)^{-\alpha-i \gamma}$ are uniformly bounded in $\alpha>0$, a corollary of the Uniform Boundedness Principle. implies that $(1+T)^{-i \gamma}=\left[(1+T)^{i \gamma}\right]^{-1}$ is a bounded operator on $L_{p}(H)$. and the desired equality holds.

COROLLARY 4.6. $J^{i \gamma}$ is the $i \gamma$-th Komatsu power of $J=J^{1}$ for all real $\gamma$.

Proof. Denote the $i \gamma$-th Komatsu power of $J$ by $(J)^{i \gamma}$. By Theorem 8.2 of [4-I], for a dense set of $f$ in $L_{p}(H),(J)^{i \gamma} f=\lim _{\alpha \rightarrow 0^{+}}(J)^{a+i \gamma} f=\lim _{\alpha \rightarrow 0^{+}} J^{\alpha+i_{\gamma}} f$ $=J^{i \gamma} f$; the second equality follows from part 4 of Theorem 1 and the strong analyticity of $(J)^{\alpha}$ and $J^{\alpha}$. Since $J^{\alpha+i \gamma}=(J)^{\alpha+i \gamma}$ are uniformly bounded in $\alpha \geqslant 0,(J)^{i \gamma}$ is bounded by the Uniform Boundedness Principle, so that $J^{i \gamma} f=(J)^{i \gamma} f$ for all $f$ in $L_{p}(H)$.

COROLLARY 4.7. For any complex number $\alpha$ in $\operatorname{Re} \alpha>0, L_{p}^{a}(H)=L_{p}^{\operatorname{Re} \alpha}(H)$, with equivalent norms. 
Proof. Let $\alpha=\beta+i \gamma$. Then because of the boundedness and invertibility of $J^{i \gamma},\left\|J^{\beta} f\right\|_{p} \leqslant K(\gamma)\left\|J^{a} f\right\|_{p} \leqslant K_{1}(\gamma)\left\|J^{\beta} f\right\|_{p}$.

Remark 4.1. The method used in Section 4 can be used to show that $J_{2}^{i \gamma}$ is a bounded operator on $L_{p}(H)$. The basic fact used above was that if

$$
\varepsilon^{U_{y}^{\alpha}} f=\Gamma(\alpha)^{-1} \int_{0}^{\infty} T_{t y} f t^{\alpha-1} e^{-t} d t+\frac{\varepsilon^{\alpha}}{\Gamma(\alpha+1)} f
$$

and if

$$
U_{y}^{\alpha} f=\Gamma(\alpha)^{-1} \int_{0}^{\infty} T_{t y} f t^{\alpha-1} e^{-t} d t \quad \text { for } \operatorname{Re} \alpha>0,
$$

then $\varepsilon^{v_{y}^{i \gamma}}$ converges strongly to a bounded operator $U_{y}^{i \gamma}$ as $\varepsilon \rightarrow 0^{+}$. Furthermore, $U_{y}^{i \gamma}$ is the strong limit of $U_{y}^{\beta+i \nu}$. Thus only minor modifications in the argument are needed to prove that $J_{2}^{i \gamma}$ is bounded. Write

$$
\begin{aligned}
J_{2}^{\alpha}(f) & =2 \Gamma(\alpha / 2)^{-1} \int_{0}^{\infty} H_{t^{2}}(f) t^{\alpha-1} \exp \left(-t^{2}\right) d t \\
& =2 \Gamma(\alpha / 2)^{-1} \int_{H} \int_{0}^{\infty} T_{t y} f t^{\alpha-1} e^{-t^{2}} d t d n \circ B^{-1}(y)
\end{aligned}
$$

$\exp \left(-t^{2}\right)$ is the Fourier transform of a bounded measure on the real line and $t^{-1}\left(\exp \left(-t^{2}\right)-1\right)$ is bounded near zero, so that

$$
\varepsilon^{J_{2}^{\alpha} f}=2 \Gamma(\alpha / 2)^{-i}\left[\int_{\varepsilon}^{\infty} H_{t^{2}}(f) t^{\alpha-1} e^{-t^{2}} d t+\frac{\varepsilon^{\alpha}}{\alpha} f\right]
$$

is uniformly bounded and converges if $\operatorname{Re} \alpha \geqslant 0$. As above $J_{2}^{i \gamma} f=$ $=\lim \left\{\varepsilon_{2}^{J_{2}^{i \nu}} f: \varepsilon \rightarrow 0^{+}\right\}$and one shows that $J_{2}^{i \gamma}=\lim _{\beta \rightarrow 0^{+}} J_{2}^{\beta+i \gamma} f$. Again $J_{2}^{i \gamma}=\left(1+T^{2}\right)^{i \eta / 2}$ and the $J_{2}^{i \gamma}$ form a strongly continuous group of bounded operators on $I_{p}(H)$ whose norm depends only on $\gamma$ and $p$. An estimate for $\left\|J_{2}^{i \nu}\right\|$ can be written easily from the estimate, given above, for $J_{1}^{i \gamma}$.

Remark 4.2. If $2 \geqslant k>0$ is a real number, one can reason as above to show that $J_{k}^{\alpha}=\left(1+T^{k}\right)^{-a / k}$ has boundary values $J_{k}^{i \gamma}$ bounded on $L_{p}(H)$. Here one has to use the special function $f_{k}(t, u)$ given in [7] to represent $\exp \left(-t T^{k}\right)$ as a semi-group given by convolution with a Borel probability measure.

5. The semi-groups $J^{\beta}$ and $J^{i \gamma}$. We shall study the spectrums and infinitesmal generators of $J^{\beta}, \beta>0$, and $J^{i \gamma}, \gamma$ real. Since $J^{\alpha}$ is an analytic semi-group with bounded boundary values, $J^{i \gamma}$, a well-known theorem in semi-group theory states that if $A$ is the infinitesmal generator of $J^{\beta}$, $\beta>0$, then $J^{i \gamma}=\exp (i \gamma A)$. In what follows $A$ denotes the infinitesmal generator of $J^{\beta}, \beta>0$.
Theorem 6. A function $f$ in $L_{p}(H)$ is in $D(A)$ if and only if

$$
f_{*}=\int_{0}^{\infty} P_{t} f e^{-t} \log t d t
$$

is in $D(T)$ when $P_{t}=\exp (-t T)$. When this is the case $A f=C f+(1+T) f_{*}$, where $C$ is Euler's constant.

Proof. Let $J=J^{1}$. If $f$ is in $D(A), A J(f)=J A(f)=\left.\frac{\partial}{\partial \beta} J^{\beta}(f)\right|_{\beta=1}$ $=-\Gamma^{\prime}(1) J(f)+\int_{0}^{\infty} P_{t}(f) e^{-t} \log t d t$. Since $J A f$ and $J f$ are in $D(T), f_{*}$ is in $D(T)$ and $A f=C f+(1+T) f_{*} ; C=-\Gamma^{\prime}(1)$ is Euler's constant $=\lim \left(\sum_{k=1}^{n} k^{-1}-\log n\right)$.

Conversely if $f_{*}$ is in $D(T)$, then

$$
\begin{aligned}
\int_{a}^{b} J^{t}\left(C f+(1+T) f_{*}\right) d t=\int_{a}^{b} J^{t}(1+T)\left(C J f+f_{*}\right) d t \\
=\left.\int_{a}^{b} J^{t}(1+T) \frac{\partial}{\partial u} J^{u}(f)\right|_{u=1} d t=(1+T) \int_{a}^{b} \frac{\partial}{\partial t} J^{t+1}(f) d t \\
=(1+T)\left[J^{b+1}(f)-J^{a+1}(f)\right] .
\end{aligned}
$$

Thus $\lim _{a \rightarrow 0^{+}} \int_{a}^{b} J^{t}\left(C f+(1+T) f_{*}\right) d t=J^{b}(f)-f$, and $f$ is in $D(A)$ since

$$
\lim _{b \rightarrow 0^{+}} b^{-1} \int_{0}^{b} J^{t}\left(C f+(1+T) f_{*}\right) d t=C f+(1+T) f_{*} .
$$

COROLLARY 5.1. The infinitesmal generator of the group $J^{i \gamma}$ acting on. $L_{p}(H)$ is $i A$, where $A f=C f+(1+T) f_{*}$ when $f_{*}=\int_{0}^{\infty} P_{t}(f) e^{-t} \log t d t$ is in the domain of $T$; $C$ is Euler's constant.

Proof. By a well-known theorem in semi-group theory, $J^{i \gamma}=$ $=\exp (i \gamma A)$ when $J^{\beta}=\exp (\beta A)$ for $\beta>0$. The properties of $A$ are given in Theorem 5 .

If $\beta>0$, the spectrum of $J^{\beta}$ is contained in the unit disk. For $J^{i \gamma}$ there is:

THeоREM 7. The spectrum of $J^{i \gamma}$ lies in the annulus $\exp (-\pi|\gamma| / 2)$ $\leqslant|\lambda| \leqslant \exp (\pi|\gamma| / 2)$.

Proof. By Theorem 4, $\left\|J^{i \gamma}\right\| \leqslant K p q(|\gamma|+1)^{2}|\dot{\Gamma}(i \gamma+1)|^{-1}$. Since $|\Gamma(i \gamma)|=\pi^{1 / 2}(\gamma \sinh \pi \gamma)^{-1 / 2}$, Corollary 4.3 implies that the spectral radius of $J^{i \gamma}$ is $r\left(J^{i \gamma}\right)=\lim _{n \rightarrow \infty}\left\|\left(J^{i \gamma}\right)^{n}\right\|^{1 / n}=\lim _{n \rightarrow \infty}\left\|J^{i \gamma n}\right\|^{1 / n} \leqslant \exp (\pi|\gamma| / 2)$. Since $J^{i \gamma}$ is invertible and since $\sigma\left(J^{i \gamma}\right)$ is a compact set in the complex plane, there 
is a real number $A$ such that $\sigma\left(J^{i \gamma}\right)$ lies in the annulus $A \leqslant|\lambda| \leqslant \exp (\pi|\gamma| / 2)$. Since $\sum_{n=0}^{\infty} \lambda^{-n-1} J^{i n \gamma}$ converges to $\left(\lambda-J^{i \gamma}\right)^{-1}$ on $|\lambda|>\exp (\pi|\gamma| / 2)$ and since $-\sum_{n=0}^{\infty} \lambda^{n} J^{-i(n+1) \gamma}$ converges to $\left(\lambda-J^{i \gamma}\right)^{-1}$ in $|\lambda|<\exp (-\pi|\gamma| / 2)$, we have the desired results.

\section{References}

[1] V. Balakrishnan Fractional powers of closed operators and the semi-groups generated by them, Pacific J. Math. 10 (1960), pp. 419-437.

[2] M. J. Fisher, Harmonic functions and their conjugates on Hilbert space, Trans. Amer. Math. Soc. 137 (1969), pp. 387-405.

[3] - Singular integrals and fractional powers of operators, ibidem (November, 1971).

[4] H. Ko matsu, Fractional powers of operators I, Pacific J. Math. 19 (1966), pp. 285-346; II. Pacific J. Math. 21 (1967), pp. 89-111; III. J. Math. Soc. Japan 21 (1969), pp. 205-220; IV. J. Math. Soc. Japan 21 (1969), 221-228.

[5] B. Muckenhoupt, On certain singular integrals, Paoific J. Math. 10 (1960), pp. 239-261.

[6] E. M. Stein, The characterization of functions arising as potentials, Bull. Amer. Math. Soc. 67 (1961).

[7] K. Yosida, Functional Analysis, Berlin 1965, pp. 259-268.

THE UNIVERSITY OF MONTANA

MISSOULA, MONTANA 59801

\section{Some remarks on the Gurarij space}

by

\section{P. WOJTASZCZYK (Warszawa)}

Abstract. Complementably universal properties of the Gurarij space of universal disposition are proved. Some linearly isomorphic equivalences between Banach spaces whose duals are $L_{1}$ spaces are stated.

A predual of $L_{1}$ is a Banach space $X$ such that $X^{*}$ is linearly isometric to $L_{1}(\mu)$ for some measure $\mu$.

DefrNition. A separable space $X$ is a space of universal disposition iff for every finite dimensional Banach spaces $F \supset E$ and every isomorphism $T: E \rightarrow X$ and every $\varepsilon>0$ there is an isomorphism $\tilde{T}: F \rightarrow X$ such that $\tilde{T} \mid E=T$ and $\|\tilde{T}\| \cdot\left\|\tilde{T}^{-1}\right\| \leqslant(1+\varepsilon)\|T\|\left\|T^{-1}\right\|$.

Such a space was first constructed by Gurarij [1] and next by Lazar and Lindenstrauss [3].

In this note we prove the following

THEOREM. Let $X$ be a separable predual of $L_{1}$. Then there exists a Banach space of universal disposition $\Gamma_{X}, \Gamma_{X} \supset X$ and there is a projection of norm one from $\Gamma_{X}$ onto $X$.

The proof of this Theorem is a slight modification of Gurarij's proof [1].

By [5], Theorem 4.2 there exists a Banach space $Y$ such that:

(*) $Y$ is a separable predual of $L_{1}$ and for any separable predual of $L_{1}$, say $X$, and any $\varepsilon>0$ there exist an embedding $T: X \rightarrow Y$, $\|T\|\left\|T^{-1}\right\| \leqslant 1+\varepsilon$ and a projection of norm one from $Y$ onto $T(X)$.

By [4] Remark e after Theorem 4 there exists a separable predual of $L_{1}$, say $W$, such that any separable predual of $L_{1}$ is a quotient space of $W$.

If we apply the above Theorem for $X=Y$ or $X=W$ we obtain

COROLLARY 1. The spaces $Y$ and $W$ can be choosen to be of universal disposition.

COROLLARY 2. Every space which satisfies $(*)$ is isomorphic to every space of universal disposition. 